\section{What about steroids?}

We recognize the desire to produce a "Five things to know about ..." article for a common clinical condition. After all, the popular press constantly barrages us with similar entertaining lists of facts we didn't know about certain things. Squissato and Brown ${ }^{1}$ have selected some interesting articles on which to comment from many thousands of possible articles. The danger of this approach was that it was completely at the discretion of the authors to select what they considered important topics and to hopefully then give an unbiased assessment of that topic. The article does not cite any of the 12 available Cochrane reviews on the topic of carpal tunnel syndrome.

For the most part, the article does a good job of simplifying the current knowledge. However, we take issue with point five regarding treatment of carpal tunnel syndrome. The authors based their recommendation on a small randomizedcontrolled trial comparing wrist splints and an educational program and a control group who received nothing. ${ }^{2}$ Perhaps not surprisingly, the control group experienced a dropout rate of over $22 \%$ compared to 3\% in the treatment group. This obviously places the internal (and therefore external) validity in question. The study ultimately went on to show an advantage to the splint group. But why include this study in the first place when there is a Cochrane systematic review published just the year before that looked at 19 studies of wrist splints with almost 1200 patients enrolled?

We have concerns about the recommendation to consult an occupational therapist for splinting. Wrist splints are available and inexpensive, and basic advice on activities to avoid is within the purview of the primary care practitioner. We suggest referral to an occupational therapist or orthotist only when over-thecounter splits don't fit well (such as carpal tunnel syndrome associated with rheumatoid arthritis) to avoid delay in initiating treatment and additional expense.

More worrisome is Squissato and Brown's ${ }^{1}$ conclusion that, "if symptoms do not improve within eight weeks, referral to a surgical specialist should be considered." There is no evidence that eight weeks of splinting is the limit. This recommendation could lead to unnecessary surgical consultations. There is no mention of electrodiagnostic studies in the diagnosis and monitoring of the condition and no mention of the one treatment that has the best evidence of efficacy in carpal tunnel syndrome, corticosteroid injection. ${ }^{4}$

\section{Nigel Ashworth MBChB MSc, Jeremy Bland MBChB, Kristine Chapman MD,} Gaetan Tardif MD

Department of Medicine (Ashworth), University of Alberta, Edmonton, Alta.; EEG Department (Bland), Kent and Canterbury Hospital, Canterbury, UK; Neuromuscular Disease Unit (Chapman), Diamond Health Care Centre, Vancouver, BC; Division of Physiatry (Tardif),

Toronto Rehabilitation Institute, Toronto, Ont

\section{References}

1. Squissato V, Brown G. Carpal tunnel syndrome. CMAJ 2014;186:853.

2. Hall B, Lee HC, Fitzgerald $\mathrm{H}$, et al. Investigating the effectiveness of full-time wrist splinting and education in the treatment of carpal tunnel syndrome: a randomized controlled trial. Am J Occup Ther 2013;67:448-59.

3. Page MJ, Massy-Westropp N, O'Connor D, et al. Splinting for carpal tunnel syndrome. Cochrane Database Syst Rev 2012;(7):CD010003.

4. Marshall S, Tardif G, Ashworth N. Local corticosteroid injection for carpal tunnel syndrome. Cochrane Database Syst Rev 2007;(2):CD001554.

Competing interests: Ashworth and Tardiff coauthored the Cochrane review on corticosteroid injection.

\section{CMAJ 2015. DOI:10.1503/cmaj.115-0001}

\section{Too much focus on low- quality science?}

The controversy concerning dietary sodium results primarily from lowquality studies and their commercial marketing and promotion. ${ }^{1-5}$ Lowquality studies do not adequately assess sodium intake, they use extreme variation in dietary sodium and they measure outcomes over a duration of a few days. They do not address known confounding factors for the outcomes being tested nor do they control for blood pressure (the main mechanism of sodium-induced harm) and they are conducted in populations with diseases where reverse causality is likely. Such poor-quality studies are often promoted and occasionally conducted by consultants of the Salt Institute (an umbrella organization of the salt industry). The publication of such studies, leveraged by the private sector, has created a false aura of scientific controversy around dietary salt. ${ }^{1-5}$

Although the call for a large randomized controlled trial on dietary sodium is not new, it has limited feasibility in Western countries where the food supply contains so much sodium. ${ }^{6}$ In Africa, where some populations still have low sodium intake, it was deemed unethical to increase dietary sodium in a trial setting. In China, where sodium added during cooking is a major source of dietary sodium, a large randomized controlled trial with a salt substitute is underway, but results will be confounded by very high baseline sodium intake and the need to use a salt substitute with potassium (a beneficial nutrient). In other countries, extensive dietary advice and support, when used alone, has proven ineffective at substantially lowering dietary sodium over the long haul. ${ }^{7}$ Hence, a large trial based on advice alone is unlikely to lower sodium intake, let alone show changes in outcomes.

It is important to also consider the World Health Organization (WHO) forum and technical meeting, "Reducing salt intake in populations," discussed by MacLeod and Cairns. ${ }^{8}$ The WHO forum was developed around controversial new evidence from the PURE study, which categorized an individual's long-term sodium intake based on a single "spot" (fasted first morning) urine sample., 910 This method is widely recognized as inadequate to assess a person's usual sodium intake, would not meet the minimum study quality criteria of blood-pressure studies for inclusion in the WHO evidence review ${ }^{11,12}$ and is therefore unlikely to have a bearing on dietary sodium recommendations. ${ }^{13,14}$

It is concerning that the PURE validation study for using spot urine samples was fraught with methodologic 